\title{
Hello from the other side: What I wish I'd done differently in training
}

\author{
Alexander Kraev, MD, ${ }^{a}$ and HelenMari Merritt-Genore, DO $^{\mathrm{b}}$
}

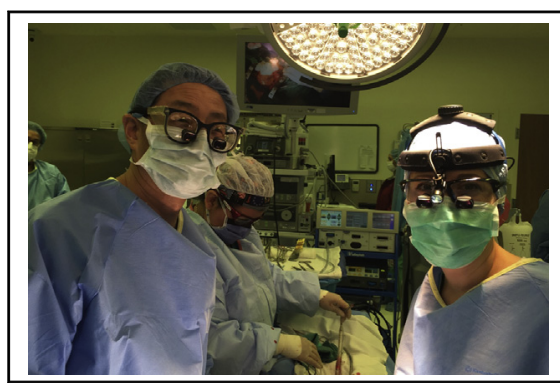

"Take time to stop and smell the BioGlue!" Photo taken on last day of training.

\section{Central Message}

Do-overs during training: "What I wished I had done differently during my training and what I wished my program had focused on more, from the perspective of an adult cardiac surgeon.'

See Editorial Commentary page 1437. Training" and have identified 4 concepts to emphasize: (1) sweat the small stuff; (2) slow down; (3) avoid the plateau; and (4) anticipate change.

\section{SWEAT THE SMALL STUFF}

Go back to the basics during your last year. You will have a renewed perspective and attention to detail as you consider your impending independence. Review sutures-braided or monofilament; absorbable versus nonabsorbable? Review needles - understand the relevance of the size, geometry, and designed purpose. For example, the taper needle is for soft tissues, and the reverse cutting goes through connective tissue. Some professors would use C-1 needle for proximal vein graft anastomosis; others would use the RB-1 needle. Another used a Gore-Tex stitch because it did not saw through tissues like Ethibond and had no memory like Prolene. The needle is also the thickness of the suture, so the holes won't bleed. The little details take years to appreciate and then to evaluate. Seek more clarification as to "why," so you'll be able to make your own decisions later.

Be aware that your new hospital may use different brands of sutures or instruments. Change your focus to understanding what type of suture or instrument you want, and this will help ease the clarification process as you start your practice. In training, you may extend your hand, and the exact item you need will appear magically by the experienced scrub tech. This is not so when you're starting your own practice and can be a difficult transition when you need a stitch quickly. Again, you will want to learn not only the names of what you need but also learn to describe accurately these tools so the team can help you more effectively. "I need a big half-circle needle, no pledget, monofilament 4-0 suture, double-loaded, forehand then backhand." Be precise. With time, those things will start to appear in your hand as well.

Review all the different types of valves commercially available. In fact, do a grand rounds presentation on it! There is a good chance you will use a valve you've never seen for your first case. Take advice from the company reps but also do your own research. You might want to consider asking for a wet laboratory with porcine hearts for practice before your first implantation. Know the pros/cons and nuances of implantation. As with everything, know the data.

You will want to build your preference cards during your chief year, or you will start with the standard sets from your new operating room, which may be missing some of your favorite "go-to" instruments. At the start of a career, it's hard to be flexible and to adapt outside of the comfort zone and rhythm you have finally settled into at the end of training. Unfortunately, there is no way around this. The first day as an attending is start of a new fellowship. Keep the phone number of your charge nurse and techs in your phone and call to get item numbers or photographs of the "must-have" instruments. Trust us, they are expecting to hear from you!

\section{SLOW DOWN}

One attending, an avid golfer often said, "Most mistakes are made before the swing." It's such a great quote, 
illustrating how preparation and mental vigilance leads to accomplishment. A successful surgery, in turn, begins with attention to even the smallest details of the case setup, and a carefully thought-out Plan A, B, and C. You must understand, and frequently be present for, the set-up of the case. From "how much flow do you want on the $\mathrm{CO}$ insufflation" to "what size femoral arterial line would you like," there are just so many details that can get glossed over. As painful as it seems, take your time, understand, and be truly involved in every part of the case: from positioning and tucking, to transferring the patient off the operating room bed at the end. Ultimately, that will become second nature, and your team will know exactly what you want.

Spend time with the perfusionists, and fully understand the pump. This is not intuitive. Create a systematic approach for cannulation, cardiac protection, de-airing, and weaning from pump for every case. It helps to write this out for clarity. During training, lead the commands and sequence of the operation as much as possible. Develop your own rhythm for the case. Know all the settings and levels your perfusion has. For a few examples, know which cardioplegia, how much vacuum, cool to which temperature, flows, etc. Like playing a guitar and singing at the same time, combining operative skills and cardiopulmonary bypass skills does not happen right off the bat, even if you are good at both individually.

Learn to be a good first assistant. This is valuable time spent, although it won't feel like it during training. Do not daze out in the cases in which you're the second-assistant. Use this time to focus on all of the tiny details that go into a big operation. Look how your attending loads their needle. Study his or her arm and body position. Pay attention to the angle of presentation for valve sutures and for conduits. This is especially important if you will be training residents and fellows. Assisting, too, is not intuitive and takes dedicated practice.

Pay particular attention to the junior surgeons and glean from them as much as you do the senior surgeons. Their struggles will soon be yours. You will be proud of navigating through your first tough case solo, only to turn around and be humbled by the next. It is good to remember in these times that, although you have moved from the shelter of residency, learning in cardiothoracic surgery is never over. You will study even more during your junior attending years than you have during training.

The Golden Rule of "Treat others as you would like to be treated" goes a long way during the stressful or exhausted moments that occur in training. Sharp words leave a lasting cut. Your integrity and work ethic will be remembered far longer than your performance on a test. Also, you never know with whom you'll be working, or for, one day. On that note, cherish the friendships you make with your co-residents, because they will likely become your closest colleagues in the field.

\section{AVOID THE PLATEAU}

Step back as you enter your last year of training. You should not be comfortable. Keep pushing yourself and pushing hard. One attending used to remind me that when she started critiquing something new, it just meant that I had mastered the last thing. It's a good perspective to keep in mind and makes the critique easier to accept.

You will want to take on more and more of the case. When you have the routine cases down, ask to have the intern or another less-experienced person as your first assist. It is important to learn to guide unfamiliar assistants to best help you. Do not take for granted the ways your attending is helping make the exposure great or showing you the conduit just right. These are subtle things that make a world of difference when they're gone.

Do not fall into the paradoxical situation of mastering the most complex surgeries while ignoring some of the "smaller cases." They will make up a good deal of your independent practice when you start. There are no junior cases once you take away the training wheels and start from scratch. Your first case may be a groin cutdown for a transcatheter aortic valve replacement. You might end up being more prepared for a complex Type A repair than for femoral artery repair! Other great examples are left ventricular lead placement, pericardial windows, pacemakers, biopsies, and permanent vascular access. Don't skip these bread-and-butter cases and be forced to reinvent them as an attending. It's helpful to remind yourself that you are not there to show the attending surgeons what you know but to soak in every bit of what they know. This is your one protected time to ask questions and make mistakes with someone there to help you.

\section{ANTICIPATE CHANGE}

The field of cardiac surgery is rapidly changing as we innovate and adapt. Get exposure in extracorporeal membrane oxygenation, thoracic endovascular aortic repair, transcatheter aortic valve replacement, ventricular assist devices, video-assisted thoracoscopic surgery/ robotics, and minimally invasive approaches. Every job will ask you about one or all of these experiences. If your program can not offer these rotations, try and set up additional electives. The exposure will serve you well whether or not you take a position that requires these proficiencies. During your time on general surgery, pay attention to the trauma, laparoscopic, and endovascular surgeries. Especially in rural practice, you will need these skills.

Understand that learning new techniques as an attending is different. You may watch a video on the key points, but when you go to observe the case, you'll find yourself focused on all the parts of a surgery that are nontechnical. The preference card, the time-out, communication between 
team members, the set-up of the room, the mayo stand, the circulator, the sequence and rhythm of the case...these all come into focus as you see the case as a whole rather than just the surgical portion. Try to adopt this vision in training.

\section{CONCLUSIONS}

Keep your eyes on the prize but remind yourself often of exactly what that prize is: not just finishing training (although that part is great!) but of emerging as a well-trained cardiothoracic surgeon, ready to enter into independent practice. Focus on every detail. Don't delegate even the smallest things. Avoid being comfortable in training. Anticipate the future of cardiothoracic surgery and hone those skills now. Most importantly, slow down. As Benjamin Franklin once said, "Lost time is never again found." 